\title{
Clinical and Histological Spectrum of Primary Cutaneous B-Cell Lymphoma in an Ethnically-Diverse Group of Patients: A Case Series
}

Sahira Farooq, BS ${ }^{1}$, Madeline Frizzell, BS, MS², Emily Limmer ${ }^{3}$, Ritu Swali, MD , Stephen Tyring, MD, PhD, MBA ${ }^{4,5}$

${ }^{1}$ McGovern Medical School, Houston, TX

2 Texas A\&M University College of Medicine, Houston, TX

${ }^{3}$ University of Texas Southwestern Medical School, Dallas, TX

${ }^{4}$ Department of Dermatology, University of Nebraska Medical Center, Omaha, NE

${ }^{5}$ Department of Dermatology, McGovern Medical School at UT Houston Health Science Center, Houston, TX

\section{ABSTRACT}

Primary cutaneous lymphomas, which are diagnosed with only skin involvement, can be of B or T cell origin. Due to their varied origin as well as their diverse presentations, these groups have been divided further into more descriptive and representative groups. The subtypes are primary cutaneous marginal zone lymphoma (PCMZL), primary cutaneous follicle center lymphoma (PCFCL), primary cutaneous diffuse large B cell lymphoma, leg type (PCDLBCL-LT) and intravascular large B-cell lymphoma. Here, we present the cases of three ethnically diverse patients with distinct clinical and histological forms of cutaneous B cell lymphoma.

\section{INTRODUCTION}

While dermatologic findings may not be what initially come to mind when identifying lymphomas, these neoplasms can and do present cutaneously. Primary cutaneous lymphomas, which are diagnosed with only skin involvement, can be of $\mathrm{B}$ or $\mathrm{T}$ cell origin, with B cells making up merely 25$30 \%$ of cases. They commonly present with nodules or with plaques or papules. ${ }^{1}$ Previously lymphomas were classified as simply Hodgkin or non-Hodgkin. ${ }^{2}$ However, due to their varied origin as well as their diverse presentations, these groups have been divided further into more descriptive and representative groups. Broadly, nonHodgkin lymphoma derived from B cells include diffuse large $B$ cell lymphoma
(DLBCL), Burkitt lymphoma, follicular lymphoma, and marginal zone lymphoma, among others. ${ }^{3}$ Here, we present the cases of three ethnically diverse patients with distinct clinical and histological forms of cutaneous B cell lymphoma.

\section{CASE PRESENTATION}

\section{Patient A}

A 67-year-old Hispanic male, with a past medical history of coronary artery disease, hypertension, hyperlipidemia, and type 2 diabetes, presented to the dermatology clinic with a two-month history of a red nodule on his left ear. He denied pain, pruritus, and/or bleeding. He had been using triamcinolone $1 \%$ ointment on the lesion with minor improvement. On clinical examination, 
there was a pearly nodule on the left antitragus (Figure 1). A shave biopsy was performed to rule out basal cell carcinoma. Histopathological examination revealed an atypical dense infiltrate of lymphocytes with a Grenz zone. Immunohistochemistry demonstrated $\mathrm{CD} 20+, \mathrm{CD} 3+, \mathrm{BCL}-2+$, CD10+, BCL-6+, and Ki67+, while MUM1-, CD30-, and CD21-. Results were consistent with germinal center type diffuse large B-cell lymphoma.

\section{Patient B}

A 79-year-old African American male, with a history of diabetes and hypertension, presented to the dermatology clinic with a scalp lesion present for the past two years. $\mathrm{He}$ noted that the lesion stung upon manipulation. No treatment had been pursued up to this point. On physical exam, there was a notable skin-colored nodule $(33 \mathrm{~mm} \times 22 \mathrm{~mm})$ on the left crown of the scalp (Figure 2). A punch biopsy was performed. Histopathology revealed sheets of markedly atypical lymphoid cells. Immunohistochemical stains were CD20+, $\mathrm{Ki}-67+, \mathrm{CD} 3+, \mathrm{CD} 4+, \mathrm{CD} 8+, \mathrm{CD} 30+, \mathrm{CD} 10-$, CD23-, S100-, AE1/3-. Lesional cells were $\mathrm{MUM}+$, consistent with diffuse large B-cell lymphoma, leg type.

\section{Patient C}

A 73-year-old Caucasian male with a past medical history of hypertension, diabetes, and anemia, presented to the dermatology clinic with a bleeding growth on his right upper extremity for the past month. Clinical examination revealed a cluster of $2-4 \mathrm{~mm}$ pink-red papules and vesicles on his right wrist (Figure 3). A shave biopsy was performed. Immunohistochemistry demonstrated CD20+, CD3+, CD4+, CD5+, CD8+, BCL-2+, CD10-, CD30-, S100-, CD21-, EBER-. The lambda/kappa ratio was elevated by in-situ hybridization. The diagnosis of marginal zone B-cell lymphoma was favored.

The patients were referred to oncology for further work up and therapy.

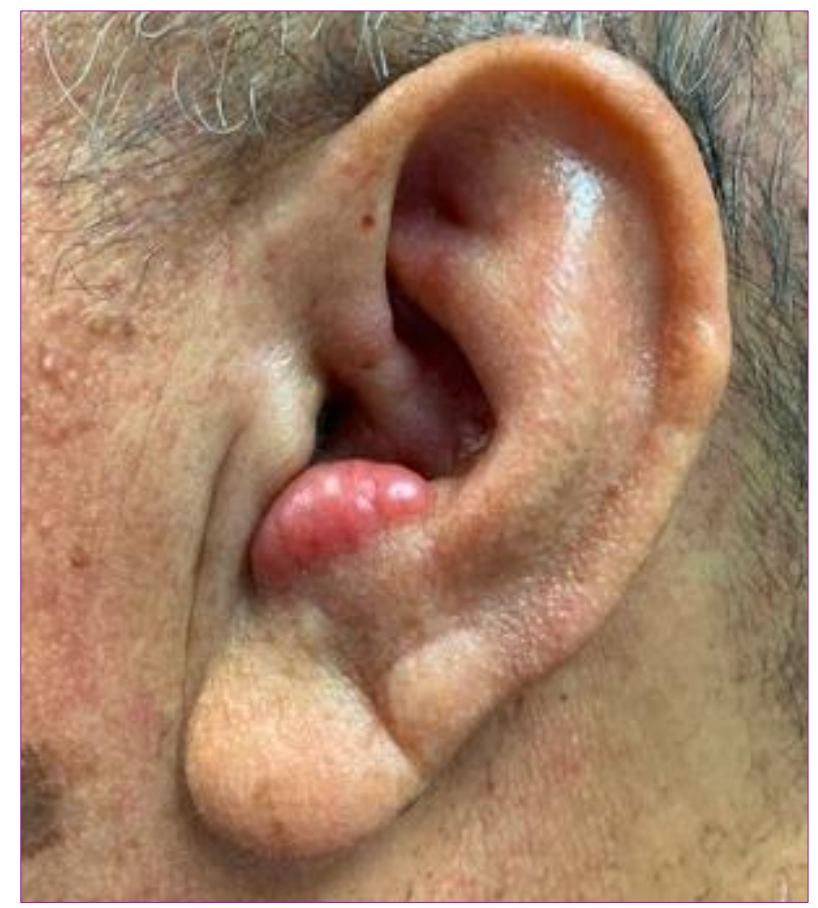

Figure 1. Patient A. A pearly nodule, reminiscent of basal cell carcinoma, representing germinal center type diffuse large B-cell lymphoma on the ear of a 67year-old Hispanic male.

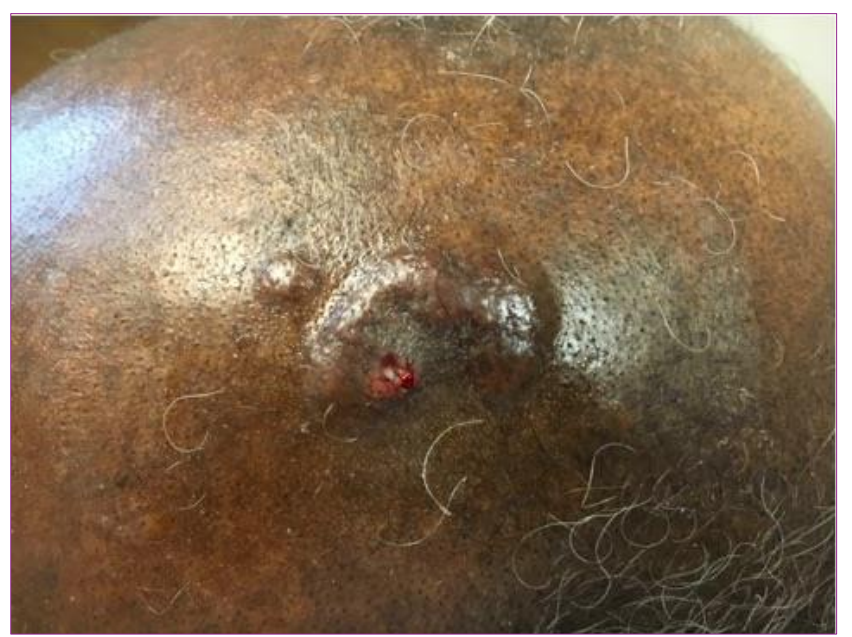

Figure 2: Patient B. Symptomatic, skin-colored lesion representing diffuse large B-cell lymphoma, leg type, on the scalp of a 79-year-old African American male. 


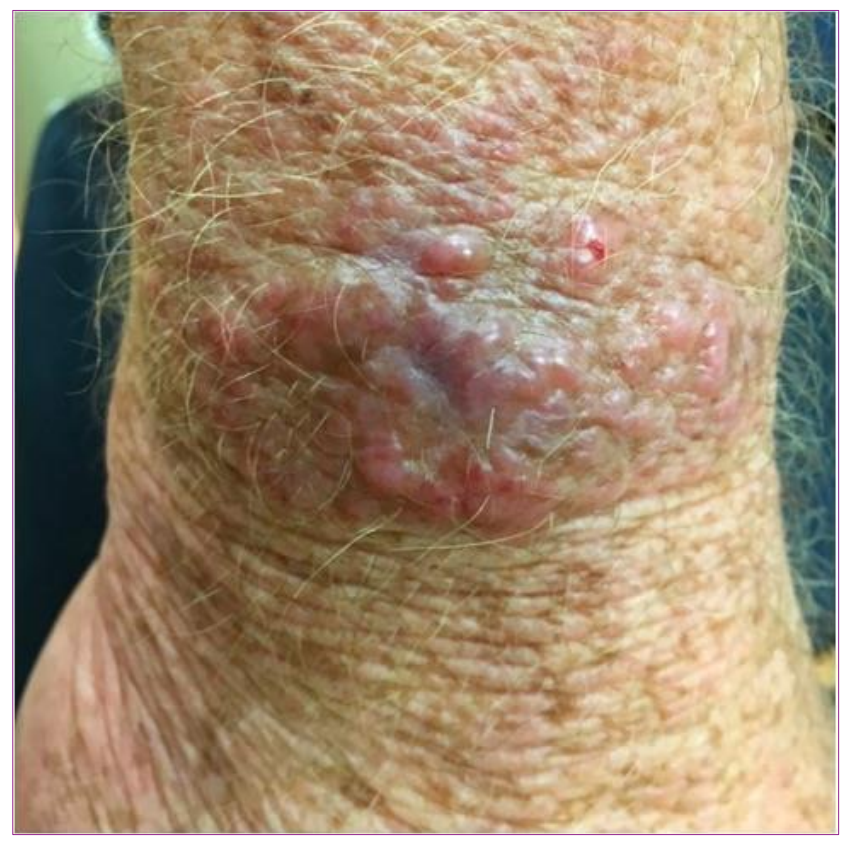

Figure 3: Patient C. Bleeding, erythematous papules and vesicles representing marginal zone B-cell lymphoma on the wrist of a 73-year-old Caucasian male.

\section{DISCUSSION}

Primary cutaneous lymphomas, which are skin-limited lymphoproliferative neoplasms or $\mathrm{B}$ or $\mathrm{T}$ cell origin, are uncommon, occurring with an estimated incidence of 0.5 to 1 case per 100,000 people annually. ${ }^{4}$ Primary cutaneous B-cell lymphomas (PCBCLs) make up around one-quarter of the cutaneous lymphomas. ${ }^{4}$

According to the World Health Organization - European Organization for Research and Treatment of Cancer (WHO-EORTC) 2018 classifications for PCL, there are 4 subtypes of PCBCLs with one provisional subtype being EBV mucocutaneous ulcer. The subtypes are primary cutaneous marginal zone lymphoma (PCMZL), primary cutaneous follicle center lymphoma (PCFCL), primary cutaneous diffuse large $B$ cell lymphoma, leg type (PCDLBCL-LT) and intravascular large B-cell lymphoma. ${ }^{5}$
Primary cutaneous lymphomas (PCLs) are, by definition, a cutaneous manifestation that is not secondary to extracutaneous spread, so their diagnosis requires a thorough workup to exclude systemic disease. A biopsy is necessary to analyze morphology, immunohistochemistry $(\mathrm{IHC})$, and staging to exclude systemic disease. ${ }^{6}$ Immunophenotype workup is also necessary to differentiate between types of cutaneous lymphomas. Presence of the CD20 marker differentiates B-cell lymphomas from T-cell lymphomas. ${ }^{9}$ Additional markers are tested to determine the pattern and location of infiltrate to distinguish between the types of cutaneous B-cell lymphomas. ${ }^{9}$ Panel recommendations include CD3, CD5, CD10, BCL2, BCL6, and MUM1. Additional testing can include CD21, CD23, CD43, Cyclin D1, $\mathrm{Ki}-67$, immunoglobulins $\operatorname{lgM}$ and $\operatorname{lgD}$, as well as kappa/lambda light chain analysis. ${ }^{4}$

WHO's current classification system of PCBCLs considers primary cutaneous diffuse large B-cell lymphoma-leg type (PCDLBCL-LT) to be the only primary cutaneous diffuse large B-cell lymphoma. While patients $A$ and $B$ were both diagnosed with PCDLBCLs because there have been multiple reports of PCDLBCL that do not fit into the leg-type description, there is still debate as to whether they represent an atypical variant of the leg type or a separate entity. ${ }^{10}$ PCDLBCL-LT commonly presents in elderly females with rapidly progressive tumors on the legs. However, $15-20 \%$ of the lesions present at locations outside the legs, such as our patient B.,6 On the other hand, PCDLBCL presents more commonly on the trunk of elderly men with frequent involvement of the limbs, head and neck regions. ${ }^{10}$ Histologically, the leg type is characterized by diffuse large cells infiltrating to subcutaneous tissue with rare intermingled reactive $T$ lymphocytes; PCDLBCL has a histologic pattern that 


\section{SKIN}

Figure 4: Primary Cutaneous Lymphoma: Stratification and Demographic Data. Stratification of primary cutaneous B-cell lymphoma into its subgroups is shown in the blue boxes. Demographic data overall and with respect to specific groups is detailed in the gray boxes.

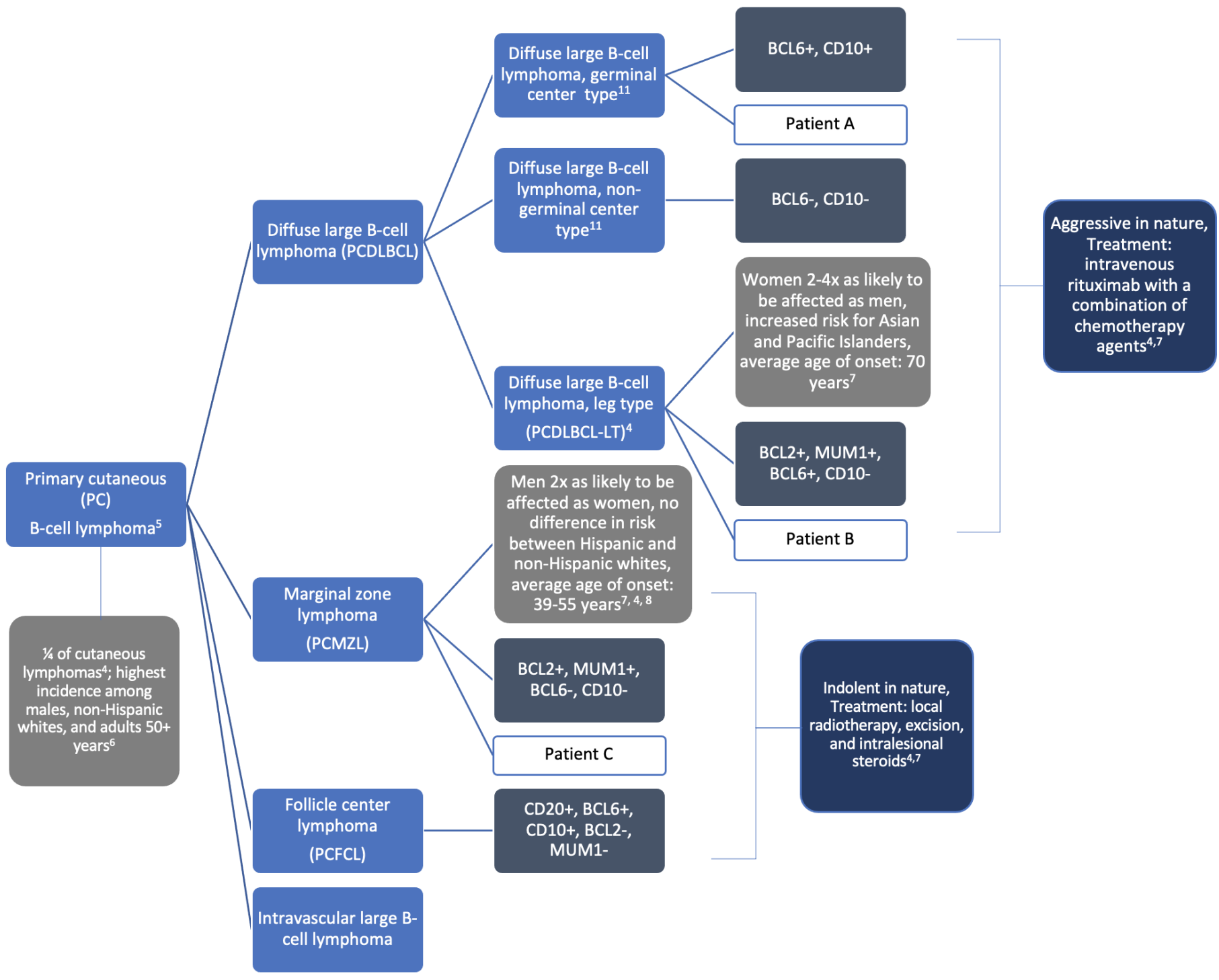

includes large centroblastic or immunoblastic cells.

Based on expression of BCL-6, CD10, and MUM-1, diffuse large b-cell lymphomas can be further divided into germinal center (GC) and non-germinal center subtypes. ${ }^{11}$ Given that BCL-6 and CD10 are markers for germinal center B-cells, patient A's IHC results were consistent with a diagnosis of PCDLBCL, GC subtype. ${ }^{11}$ Patient's B's IHC results were consistent with a diagnosis of PCDLBCL-LT, as leg type is usually positive for BCL2, MUM1 and BCL6, but negative for CD10. ${ }^{4}$

Primary cutaneous marginal zone lymphomas (PCMZL) is an indolent lymphoma, classically presenting as solitary or multifocal erythematous to brown-colored plaques, papules, or nodules. They typically appear in young adults distributed on the 
trunk or upper extremities. ${ }^{4,5}$ Pruritus is commonly associated with PCMZL, but pain, night sweats, fever and weight loss are not expected. ${ }^{5}$ On histopathology, PCMZL reveals a nodular or diffuse mixed infiltrate of small, marginal zone B-cells, lymphoplasmacytic cells, plasma cells, and reactive $T$ cells. These findings typically spare the epidermis and superficial papillary dermis. Marginal zone $B$ cells are characteristically BCL-2 and MUM-1 positive, but lack BCL-6 or CD10 expression. 4,6 The immunohistochemistry features of Patient C's biopsy and the characteristics of the tumor on histopathology favored a diagnosis of PCMZL.

Another PCBCL worth mentioning, although not found in our three patients, is primary cutaneous follicle center $B$ cell (PCFCL). The median age of presentation is 60 and typically presents with localized skin lesions on the head, neck and trunk. ${ }^{4}$ PCFCL is characterized by a predominance of large centrocytes and centroblasts that stain positive for CD20, BCL-6, CD10 and negative for BCL-2 and MUM-1. This lymphoma has an excellent prognosis and can be easily managed with local radiotherapy. ${ }^{5}$

There are limited trials and data regarding treatment for PCBCLs, and therapy is primarily guided by whether the nature of the lymphoma is indolent or aggressive. ${ }^{4}$ No standard of treatment exists for the indolent lymphomas, such as PCMZL and PCFCL, however, treatment options include local radiotherapy, excision, and intralesional steroids. ${ }^{4,7}$ Prognosis is excellent with 5year survival of $\geq 95 \% .4,7$ In contrast, PCDLBCL is aggressive in nature, and first line treatment is intravenous rituximab with a combination of chemotherapy agents. ${ }^{4,7}$ Prognosis is poorer with 5-year survival of $50-70 \% .4,7$ Relapses and systemic spread are common for PCDLBCL despite therapy. ${ }^{7}$

\section{CONCLUSION}

Overall, though PCBCLs are uncommon, it is important for clinicians to be aware of the diverse clinical and histological presentations of these indolent lymphomas, because they have a relatively good prognosis with appropriate therapy. The more aggressive, leg type lymphoma must be closely managed given its poorer prognosis. Optimal care of PCBCLs may involve a multidisciplinary approach, consisting of dermatology, medical oncology, and radiation oncology. ${ }^{6}$

\section{Conflict of Interest Disclosures: None}

Funding: None

Corresponding Author:

Ritu Swali, MD

985645 Nebraska Medical Center

Omaha, NE, 68198

Phone: 402-552-5525

Email: rituswali@gmail.com

\section{References:}

1. Kempf W, Kazakov DW, Mitteldorf C. Cutaneous lymphomas: an update. Part 2: B-cell lymphomas and related conditions. Am J Dermatopathol, 2014. 36(3): p. 197-208; quiz 209-10.

2. Alizadeh $A A$, et al. Distinct types of diffuse large B-cell lymphoma identified by gene expression profiling. Nature. 2000. 403(6769): p. 503-11.

3. Teras, LR, et al., 2016 US lymphoid malignancy statistics by World Health Organization subtypes. CA Cancer J Clin. 2016. 66(6): p. 443-459.

4. Malachowski SJ, Sun J, Chen P-L, SeminarioVidal L. Diagnosis and Management of Cutaneous B-Cell Lymphomas. Dermatologic Clinics. 2019;37(4):443-454.

5. Willemze R, Cerroni L, Kempf W, Berti E, Facchetti F, Steven H. Swerdlow, Elaine S. Jaffe; The 2018 update of the WHO-EORTC classification for primary cutaneous lymphomas. Blood. 2019; 133 (16): 1703-1714. March 2021 Volume 5 Issue 2 
6. Campbell SM, Peters SB, Zirwas MJ, Wong HK, Campbell SM. Immunophenotypic diagnosis of primary cutaneous lymphomas: A review for the practicing dermatologist. J Clin Aesthet Dermatol. 2010;3(10):21-25.

7. Sica A, Vitiello $P$, Caccavale $S$, et al. Primary Cutaneous DLBCL Non-GCB Type: Challenges of a Rare Case. Open Med (Wars). 2020;15:119125. Published 2020 Mar 19.

8. Bradford PT, Devesa SS, Anderson WF, Toro JR. Cutaneous lymphoma incidence patterns in the United States: a population-based study of 3884 cases. Blood. 2009;113(21):5064-5073.

9. Wilcox RA (2013), Cutaneous B-cell lymphomas: 2013 update on diagnosis, risk-stratification, and management. Am. J. Hematol., 88: 73-76.

10. Hans CP, Weisenburger DD, Greiner TC, et al. Confirmation of the molecular classification of diffuse large B-cell lymphoma by immunohistochemistry using a tissue microarray. Blood. 2004;103(1):275-282.

11. Goyal A, Leblanc RE, Carter JB. Cutaneous Bcell lymphoma. Hematol Oncol Clin North Am. 2019;33(1):149-161. 